\title{
Determine the best Boundary Shape of Toll Plaza
}

\author{
Yu Lv \\ North China Electric Power University, Beijing, 102206 \\ alanncepu@foxmail.com
}

Keywords: Effectiveness; Shape; Multi-objective programming; Optimization

\begin{abstract}
With the socio-economic development, people's travel rate has greatly improved. Congestion problems and security issues of High-speed junction toll station have become increasingly prominent.

A barrier toll is a row of tollbooths placed across the highway, which charges for the vehicles. A toll plaza covers the area of fan-in zone, fan-out zone and the toll barrier itself, aiming at facilitating the fee collection procedure. The shape of the barrier toll and the layout of it will influence the throughput, accident prevention and cost to a great extent.

This paper tries to design the most optimal toll plaza by using the model of Multi - objective programming taking toll plaza's shape, size and merging pattern as planning objectives.

\section{Introduction}

As the number of vehicles surge, people's travel rate increases, the highway toll port traffic pressure is increasing. By now, there have been many traffic congestion and traffic accidents around the world, which have serious consequences. The shape, size, and merging pattern of a toll station largely affect its capacity, throughput, incident prevention, and cost, all of which must be taken into account when designing toll stations. In order to achieve the maximum capacity and accident prevention capability on the basis of the lowest cost, it is necessary to design the toll station reasonably. To this end, this paper introduces a multi-objective programming model with shape, size and merging pattern, which aims to solve the problem of determining the best boundary shape of toll plaza.
\end{abstract}

\section{Establish a Road Model}

Generally, a toll highway is a two-way road. While the inside lane of each way is linear, the outside lane is streamline, which is wide in the middle part and narrow on both ends. There are $L$ lanes in each direction and a barrier toll containing B tollbooths and $B+1$ tollbooth egress lanes.

The design of a toll plaza's size and shape focuses on the shape of the outside lane of the fan-in area and fan-out area. As for the design of the merging pattern, we will set the rules for merging vehicles. Based on the assumption that fan-in area and fan-out area are image symmetrical with respect to the barrier toll, we take out the fan-out area and create the coordinate system on this part. (Fig. 1)

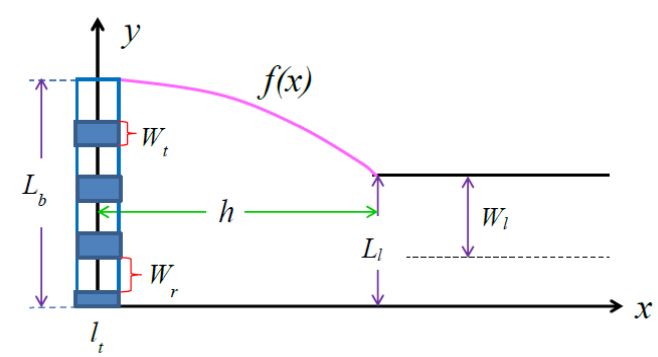

Figure 1. Road coordinate system

Considering that the road wouldn't have sudden curve nor a sharp corner on the highway, we establish boundary shape function $F(x)$ in linear function, quadratic function and exponential 
function as the pink curve indicates in Fig. 1. As long as the function expression is confirmed, the corresponding shape and size of the toll plaza can be confirmed.

\section{Model Construction}

This is a multi-objective programming problem. The shape and size of the toll plaza are determined by function $f(x)$. We have designed the practical merging pattern in advance because it's hard to quantize merging pattern into parameter. Under each merging pattern, we compute the optimal coefficient of each function $f(x)$, and then choose the optimal merging pattern through comparison analysis.

The Establishment of Objective Function for Merging Pattern. Drivers always tend to choose the shortest route, so in the first merging pattern, during the process of driving from the larger number of tollbooth egress lanes to the smaller number of out-coming lanes, the probability of choosing one lane is in inverse proportion of the length of this route. Hence, we use matrix $P_{i j}$ to represent the probability of the $i$ th(the value of $i$ is bigger on the outer lane) incoming lane to the $j$ th(the value of $j$ is bigger on the outer lane) tollbooth.

$$
P_{i j}=\frac{1}{\sqrt{(i-j-1) \times\left(W_{t}+W_{r}\right)^{2}+h^{2}}}
$$

The probability of the $j$ th tollbooth receiving vehicles can be expressed as $P_{j}$

$$
P_{j}=\sum_{i=1}^{i=L} P i j
$$

Matrix $Q_{j i}$ represents the probability of the $j$ th tollbooth to the $i$ th out-coming lane. So $Q_{j i}$ can be expressed as

$$
Q_{j i}=P_{j} \cdot P_{i j}
$$

We define the variance distribution of $Q_{j i}$ as the coefficient factor $\lambda_{i}$ of each out-coming lane, and thus $\lambda=\sum_{i=1}^{i=L} \lambda_{i}$ will act on throughput of the toll plaza as the coefficient factor.

The second merging pattern is based on big data analysis theory. We consider the probability that each tollbooth being chosen meets normal distribution. Tollbooths in the middle stand a larger chance of being chosen and tollbooths on the sides are less likely to be selected. So the probability that the ith tollbooth being selected is

$$
P_{i}=\frac{1}{\sqrt{2 \pi} \sigma} \int_{0}^{i} e^{-\frac{(i-\mu)^{2}}{2 \sigma^{2}} d i}
$$

$\sigma$ reflects the willingness of vehicles driving towards the tollbooths in the middle. The probability that each tollbooth is chosen fluctuated round $\mu$.

Generally speaking, the faster the vehicles run, the better highway capacity is. Here we have the expression of throughput (denoted by variable $C_{b}$ )

$$
C_{b}=(9 v+1270) \times \lambda
$$

We roughly divide vehicles on road into oversize, middle-sized and small-sized vehicles. The proportion of them on the highway is $p_{b}, p_{m}, p_{s}$, and $100 \mathrm{~km}$ acceleration is $3 \mathrm{~m}^{2} / \mathrm{s}, 7.6 \mathrm{~m}^{2} / \mathrm{s}, 10 \mathrm{~m}^{2} / \mathrm{s}$ respectively. The expression of average acceleration $a_{v}$ is

$$
a_{v}=3 \times p_{b}+7.6 \times p_{m}+10 \times p_{s}
$$

We roughly define $h$ as the driving distance. From $v^{2}=2 a h$, we have 


$$
v=\sqrt{2 a h}
$$

The Establishment of Objective Function for Cost. Here we only take the accommodation cost of the toll plaza into consideration, leaving out the cost of tollbooth. So the objective function for cost (denoted as $C_{t}$ ) is the function only relating to the floor space $(S)$.

$C_{t}=C_{u} \cdot S=C_{u} \cdot \int_{1}^{h} f(x) d x$

$C_{u}$ in the function is the unit production cost.

The Establishment of Objective Function for Accident Prevention. The road accident rate is related to the curvature of the curve $(\mathrm{K})$. Relational expression of accident rate $\left(C_{R}\right)$ and curvature $(K)$ is

$$
C_{R}=1.148 \times e^{0.1438 K}
$$

The ability to prevent accidents (denoted as $A_{p}$ ) is defined as

$$
A_{p}=\frac{1}{C_{R}}
$$

Multi-objective Programming. Three objective functions have different weights. We assume the weight of throughput is 0.6 , the weight of cost is 0.25 and accident prevention's weight is 0.15 . In order to achieve optimal values of all three objective functions, we define deviation function $J$ as

$$
J=\sqrt{\left(\frac{C_{b}}{C_{b \max }}-\max \left(C_{b}\right)\right)^{2} \times 0.6+\left(\frac{C_{t}}{C_{t \max }}-\max \left(C_{t}\right)\right)^{2} \times 0.25+\left(\frac{A_{p}}{A_{p \max }}-\min \left(A_{p}\right)\right)^{2} \times 0.15}
$$

Then objective function becomes $\min J$ then, and the constraint is

$$
\left\{\begin{array}{l}
a+b+c=L_{b} \\
a h^{2}+b h+c=L_{l} \\
a \geq 0
\end{array}\right.
$$

Function $f(x)$ is a quadratic function. The value of $\lambda$ is determined by $a, b$ and $c$. And $a, b$ and $c$ in turn determine the value of $\lambda$. So the solving process turns into a cycle solution. $\lambda$ will be given an initial value at first.

\begin{tabular}{|c|c|c|c|c|c|c|c|c|c|}
\hline $\begin{array}{r}\text { Inter } \\
\text { act-ions }\end{array}$ & $C_{b}$ & $C_{t}$ & $A_{p}$ & $\frac{C_{b}}{C_{b \max }}$ & $\frac{C_{t}}{C_{t_{\max }}}$ & $\frac{A_{p}}{A_{p \max }}$ & $a$ & $b$ & $c$ \\
\hline \multirow[t]{2}{*}{0} & 5297. & 0.70 & 307. & 1.0 & 0.49 & 1.19 & -0.0 & 0.00 & 51. \\
\hline & 629 & 31 & 96 & 53 & 25 & 9 & 051 & 6 & 85 \\
\hline \multirow[t]{2}{*}{1} & 5936. & 0.70 & 307. & 1.0 & 0.49 & 1.19 & -0.0 & 0.00 & 51. \\
\hline & 460 & 31 & 96 & 40 & 25 & 9 & 044 & 51 & 85 \\
\hline \multirow[t]{2}{*}{2} & 5937. & 0.70 & 307. & 1.0 & 0.49 & 1.19 & -0.0 & 0.00 & 51. \\
\hline & 577 & 31 & 96 & 33 & 25 & 9 & 035 & 36 & 85 \\
\hline
\end{tabular}

Model Solving. Now we take a specific circumstance as an example. We assume $L=4, B=10$. Under the first merging pattern, as for $f(x)=a x^{2}+b x+c$, when initial value $\lambda=0.85$, iteration's results are as follows in the table:

Table 1 Iteration parameter

The curve of function $f(x)=-0.0051 x^{2}+0.006 x+51.85$ can connect with the lines at the both ends smoothly, which means the curve we get is ideal. 
As for the second kind of merging pattern, we get $\lambda=0.9357$, and normalized value $\frac{C_{b}}{C_{b \max }}=1.027 \quad, \quad \frac{C_{t}}{C_{t \max }}=0.4925 \quad, \quad \frac{C_{t}}{C_{t \max }}=1.199 \quad, \quad$ and $\quad$ optimal solution $a=0.0026, b=-0.673, c=58.8204$. When calculating $f(x)=a e^{-b x}+c$, we find that $a, b$ and $c$ is plural, which doesn't accord to facts, so we reject this one.

Hence, we choose boundary function as $f(x)=-0.0051 x^{2}+0.006 x+51.85$.

\section{Conclusion}

Using Multi-objective programming, considering shape, cost, merging pattern as a factor, the best shape of toll plaza can be determined. In particular, for the case of $L=4, B=10$, the boundary curve is $f(x)=-0.0051 x^{2}+0.006 x+51.85$

\section{References}

[1] Zhou Gang. Analysis of influencing factors on freeway traffic safety and fuzzy comprehensive evaluation. [D]. Shanxi: Xi'an Jiaotong University.2004.

[2] Cheng Junlong. Study of Capacity for ETC and MTC Hybrid Toll Station. [D].Sichuang: Southwest Jiaotong University.2012.

[3] Li Xiansheng. Research on the Relation between Road Alignment and Traffic Accidents. [D].Jilin: Jin Lin University.2006.

[4] Zhong Liande. The relationship between the speed difference of large or small vehicle and the accident rate of expressway. [J].Journal of Beijing University of Technology, 2007.2, 33(2):186-188.

[5] Liao Gu. Analysis on the Capacity of Expressway Toll Station. [J].Highway Engineering.2010.6.35 (3):153-155.

[6] Zhang Xiaoming. Study of Relationship between road alignment and accident rate along with induction factors on freeway. Heilongjiang: Harbin Institute of Technology.2012.

[7] Qiao Jiangang. Study on the Change of Passenger Pilots in Expressway. [J].China Safety Science Journal.2015.11.25 (11):92-98.

[8] Liu L, Weng J, Rong J. Simulation Based Mixed ETC/MTC Freeway Toll Station Capacity[C]// 19th ITS World Congress. 2012.

[9] Shen L, Dong Y. Coordinated Control between Two Toll Booths Based on Multi-point Toll Collection [J]. Journal of Highway \& Transportation Research \& Development, 2011.

[10] Nematollahi M, Hosseini-Motlagh S M, Heydari J. Coordination of social responsibility and order quantity in a two-echelon supply chain: A collaborative decision-making perspective[J]. International Journal of Production Economics, 2017, 184:107-121. 